\title{
EVALUATION OF ESCHERICHIA COLI INFECTION CONTROL AS THE MOST PREVALENT ISOLATE AFFECTING SPERM PARAMETERS.
}

\author{
A.A Farrag*, M.M.A.Sherif**, B. M.Harron* and A.S.Abo zaid \\ Departments of *Botany and Microbiology Faculty Of Science and. **Medical Microbiology and Immunology \\ Faculty of Medicine, Al Azhar University Cairo, Egypt
}

\begin{abstract}
Genital tract infection and inflammation have been associated to 8-35\% of male infertility cases. E. coli causes agglutination of human spermatozoa and thus, leading to infertility. This study aim to evaluate if the negative influence of Escherichia coli on the motility of human spermatozoa is a consequence of E. coli adhesion or some soluble factor from E. coli are involved. Highly motile preparation of spermatozoa from normozoospermic patients were obtained by 'swim up' procedure the. Study was carried out in three parts. Motility parameters were analysed by light microscopy and Nomarski differential interference contrast microscopy directly, and after $30 \mathrm{~min} ; 2,4,6 \mathrm{hs}$ and overnight after inoculation. In The second series of experiments, bacterial replication was inhibited by addition of five antibiotics amoxicillin, ciprofloxacin, erythromycin, gentamycin and penicillin $G$. The effect of $E$. coli culture filtrates on sperm motility was investigated.Finally In a third series, E. coli growth was suppressed by heating at high temperature $(100 \mathrm{oC})$ for $30 \mathrm{~min}$. E. coli decreased the sperm motility and viability by agglutination immediately after inoculation and effect increases as the time of incubation Period increases.Amazingly, both culture media in which E. coli growth was inhibited by either (antibiotic and heating), was not able to cause any change in motility and viability. Further more, completion of incubation at Period up to 6 hs the percentage of motile spermatozoa left is $11 \%$ that is very low as compared to control. This shows that E. coli may be carrying some adhesion sites through which it binds to human spermatozoa and causes agglutination. And hence infartility
\end{abstract}

Key words: Infection control, E. Coli, Sperm parameters, Male fertility Human

\section{INTRODUCTION}

There is difference as to the influence of certain microbial infection on male infertility. Several investigators have reported difference types of microorganisms in seminal fluid (Ajabor et al. 1999). It was reported that detection of bacteria in semen does not essentially suggest infection because bacterial isolates in seminal fluid may signify colonization of the urethral, contamination, or infection. Enterobacteriaceae, Chlamydia, Ureaplasma and some gram positive bacteria are the most frequently isolated organisms in industrialized countries (Keck et al. 1998).

In some parts of the world, oligospermia and azoospermia are most common causes of male infertility which has been reported due to bacterial infections (Ajabor et al. 1999 \& Megafu 2007). Urinary tract infections are common in men, and clinicians working with infertility frequently encounter patients with these diseases. Infections include either cystourethritis, caused by trivial urinary bacteria or by sexually trans- mitted pathogens affecting fertility. The possible relationship between infection and infertility has been the subject of controversy since the second half of the 1970, and several therapeutic trials have been initiated since then. The criteria for infection-associated infertility have been laid down in the World Health Organization (WHO) manuals, and several studies of the pathogenesis of reproductive disturbance in infected men have been published in the past decade (Rowe 2000). An understanding of the link between infection of the 'accessory sex glands' and reduced male fertility has been scientifically acquired and diagnostic tools are available, but the results of antibiotic treatment in terms of fertility remain disappointing. The last is probably due to the irreversibility of functional damage caused by chronic infection/inflammation. Therefore, prevention, early diagnosis and correct treatment of infections of the male tract, both trivial and sexually transmitted, are of pivotal importance (Sergio et al. 2007). According to (WHO), seminal fluid infection was 
defined as the presence of significant bacteriospermia ( $\geq 103$ bacteria/ml ejaculate), detection of Neisseria gonorrhoeae, C. trachomatis, U. urealyticum; significant leukocytospermia (10 6 peroxidase positive leukocyte/ml ejaculate). It therefore follows that if some or all the conditions above are not met, the isolation of bacteria in semen are often regarded as contaminants by most practitioners (Okon et al. 2005). Infection is a powerful mechanism that can lead to sperm damage, deformity and eventually, male infertility. Genital tract infection and inflammation have been associated to $8-35 \%$ of male infertility cases (Askienazy-Elbhar, 2005). E. coli is the most important pathogen causing prostatitis and epididymitis (Weidner et al., 1991), but is also found in semen samples of infertile men (Dahlberg 1976) without leukospermia and clinical signs of infection (Toth and Lesser, 1981).

Among the most common microorganisms involved in sexually transmitted infections, interfering with male fertility, there are the Chlamydia trachomatis and Neisseria gonorrhoeae (Rose and Scott, 1998). Sperm motility may also be inhibited by direct effects of the bacteria on spermatozoa, for example bacterial adhesion (Wolff et al., 1993) or cytotoxins (Paulson et al., 1997), or, more indirectly, by noxious effects of the induced inflammatory process, for example polymorphonuclear leukocytes (Wolff, et al., 1990), macrophages and their toxic secretory products (Hill et al.,1987). It has been found that $U$. urealyticum can attach massively to sperms, especially at the mid-piece, thus producing marked hydrodynamic drag on infested sperm (O'Leary,1990). Colony of Candida albicans can cause sperm clumping. $50 \%$ of the sperm in the specimen undergo clumping, thereby greatly reducing the effective sperm motile count (Tian, et al.,2005).

Mycoplasma genitalium was demonstrated to adhere to all parts of the spermatozoa. Numerous M. genitalium cells bound to the sper- matozoa were shown to cause sperm agglutination. Inhibition of sperm motility has also been documented for other human and animal Mycoplasmas (Panangala, et al., 1981).

The aim of this work was to study the effect of E. coli on sperm motility and viability. We conducted some series of experiments in order to determine whether decrease in motility was associated with adhesion of E. coli to sperm or some soluble factors causes the above said changes.

\section{MATERIALS AND METHODS}

Five hundred and seminal fluid specimens from men were investigated for infertility over a period of 9 months. These seminal fluids of patients submitted to the laboratory from the fertility clinics of Hospital and outer clinics the practical work was done and The Specialist Medical laboratory". Each specimen was collected by patient himself into sterile bottle. The subjects were instructed on how to collect the specimens and submit to the laboratory within one hour of collection. They were told to first pass urine and then wash their hands and penis with soap, then rinse with water prior to masturbation and ejaculation into sterile container. The semen was collected after the patient had abstained from coitus for at least three days. The semen was them cultured on Nutrient, MacConky, Mannitol salt, Thayer martin, Eosin methylene blue, blood, kligler, peptone water, MR-VP, citrate, and chocolate agar media then incubated for 24-48 hours at $37 \mathrm{oC}$, these media used for Streptococcus pyogenes, Staphylococcus aureus, Staphylococcus epidermidis, Staphylococcus saprophyticus, Eschirishia coli, Proteus mirabilis, Proteus vulgaris, Klebseilla pneumoniae, Pseudomonas aeuroginosa, Neisseria gonorrhoeae. Also to confirm the culture results we used Microbial Identification (Cheesbrough M.1984,2007)

The infective microorganisms were identified by Gram stain and cultivation on media for 
Evaluation of Escherichia coli infection control as the most

the cultivable microorganisms, The sperm density, volume, viscosity (liquefaction), the percentage of actively motile sperms, the percentage of abnormal forms, the presence or absence of pus cells were assessed. Analysis was carried out immediately they were received (Cheesbrough M.1984,2007)

\section{Sperm preparation and incubation}

Semen samples were retrieved from 30 healthy donors with a normal sperm count $(>20 \times 10 \quad 6$ spermatozoa/ml (W.H.O.1992). The semen samples were kept at room temperature, in darkness and for no longer than $3 \mathrm{~h}$ before use. The spermatozoa were purified by a 'swim-up' procedure. Only ejaculates with normal sperm concentration and forward motility were used for further investigation. Spermatozoa were washed in HAM'S F-10 medium and centrifuged at $400 \mathrm{~g}$ for $10 \mathrm{~min}$. After removing the washing solution, the pellet containing the cells was overlayed with $1 \mathrm{ml}$ Ham's F-10 medium prewarmed to $37 \mathrm{oC}$ and incubated for $30 \mathrm{~min}$ at $37 \mathrm{oC}$. After incubation, the liquid phase was isolated and stored in a sterile vial. This 'swim-up technique' was performed in all experiments to achieve a purified sperm suspension of high motility and to prevent potential antimicrobial activity of seminal plasma (Mardh, 1975) in further investigations. This suspension was diluted to a sperm concentration of $20 \mathrm{million} / \mathrm{ml}$ with culture medium to standardize the experiment and to establish exact sperm/bacteria ratios. After dividing the suspension into five fractions, four were inoculated with a suspension of E. coli in Ham's F10 , and one was inoculated with sterile medium as control. (Cheesbrough M.1984,2007)

\section{Microorganism}

E. coli used in the present study was prepared from laboratory cultures that have been isolated as described by(Prabha et al 2009). The culture of E. coli was initially isolated from a clinical source (semen samples from patients).
Experimental Design For detection of E.Coli on Sperm quality.

Three experiments were performed. Experiment 1: incubation was performed in Luria broth (Hi-Media Laboratories, India) medium. When motility was measured, bacterial concentration was also determined in the Cellometer ${ }^{\mathrm{TM}}$ counting chamber, to evaluate the bacterial growth in each sample. Experiment 2: To prevent bacterial growth effectively five antibiotics were used in present study Amoxicillin, Ciprofloxacin, Erythromycin, Gentamicin and Penicillin G (Sigma Chemical Co., USA). Stock solutions $(1 \mathrm{mg} / \mathrm{ml})$ of each antibiotic were prepared in sterile distilled water as recommended by the manufacturer. MBC (Minimal bactericidal concentration) for each antibiotic was obtained using the standard protocol (Tian Y.H., et al 2005 ). MBC was defined as a $99.99 \%$ reduction of cell viability with respect to that of initial inoculums. In time-kill studies, the MBC is the minimal amount of antibiotic that results in a $\geq 99.9 \%$ decrease in the initial inoculums within $24 \mathrm{~h}$ in a standard test. Other conditions of incubation remained the same as in the first experiment. A total of 15 single experiments were undertaken. Filtrates of E. coli cultures were obtained by filtering an E. coli suspension grown in Luria broth for $48 \mathrm{~h}$ at $37 \mathrm{oC}$, through a Millipore filter $(0.22 \mu \mathrm{m})$. This was done to establish whether a soluble factor from E. coli might influence sperm motility. Experiment 3: This was performed same as experiment 2 except the growth of E. coli was stopped by heating at $100 \mathrm{oC}$ for $30 \mathrm{~min}$. After completion of incubation the absence of viability was confirmed by plating on nutrient agar plates. A highly concentrated suspension of E. coli served as a positive control, pure Luria broth as a negative control. Filtrates of E. coli cultures were mixed 1: 1 with sperm suspensions. Besides these investigations on motility, morphological changes in spermatozoa induced by E. coli were also examined. Morphological 
studies were undertaken using light microscopy and Nomarski differential interference contrast microscopy.

\section{Light microscopy and Nomarski differential interference contrast microscopy (NM):}

The sperm and E. coli were mixed 1:1 (v/v), and examined by light microscopy using $\times 10$ objectives immediately, $30 \mathrm{~min}, 2,4,6 \mathrm{~h}$ and after overnight incubation at room temperature. For studies using NM, the sperm- E. coli suspension was incubated at $37{ }^{\circ} \mathrm{C}$ and studied after $5 \mathrm{~min}, 30 \mathrm{~min}, 1 \mathrm{~h}, 2 \mathrm{~h}, 6 \mathrm{~h}$ and overnight incubation. At each time point, an aliquot of 3 $\mu \mathrm{l}$ was mounted on slides, and after a few minutes the sperm stuck to the glass coverslips. A total of $3 \times 100$ spermatozoa were counted and the number of sperm with E. coli attached was determined. Assessment of sperm viability by Eosin-Nigrosine:

The viability of agglutinated spermatozoa at each time-point was determined using eosinnigrosine. Briefly, two drops of $1.0 \%$ eosin were added and mixed well. After $30 \mathrm{sec}$ three drops of $10 \%$ nigrosine were added and mixed. The mixture was smeared and allowed to air dry on a clean glass slide. The slide was observed under a light microscope at X100 to differentiate the unstained live cells from the pink staine

dead spermatozoa. At least 20 fields were read for each time interval and average values were noted (Cheesbrough M.1984,2007)

\section{RESULTS}

Five hundred and seminal fluid specimens from men were investigated for infertility over a period of 9 months. These seminal fluids of patients submitted to the laboratory from the fertility clinics of hospital and outer clinics, the practical work was done and The Specialist Medical laboratory". Each specimen was collected by patient himself into sterile bottle. The subjects were instructed on how to collect the specimens and submit to the laboratory within one hour of collection. They were told to first pass urine and then wash their hands and penis with soap, then rinse with water prior to masturbation and ejaculation into sterile container. The semen was collected after the patient had abstained from coitus for at least three days.and selected (265 Sample From 500 ) by excluding samples which have the some cultures bacterial Similar Samples . (Cheesbrough M.1984,2007)

Examination of seminal fluid 500 sample (age 15-60) and select 265 random sample measurement of volume $(0.5 \mathrm{ml}-10.0 \mathrm{ml})$ different volume according to present seminal fluid and measurement viscosity at $30 \mathrm{oC}$, volume, $\mathrm{pH}$ and examination of a wet preparation to estimate the percentage of motil spermatozoa, report type of motility and the percentage of viable forms, look for cells (pus cells, R.B.Cs, spermatogenic cells and bacteria, total count ( $\mathrm{ml}$, viability and abnormal sperm count, variable count and examination of stained preparation to estimate the percentage of spermatozoa with normal morphology of spermatozoa under microscopic for detect normal and abnormal spermatozoa.

Results showing that different total count, volume, viscosity at $30 \mathrm{oC}$, pus cells, bacteria deed, RBCs, spermatogenic cells/ H.P.F and different viability (active, sluggish, abnormal forms count and classification according to sperm count $/ \mathrm{ml}$ through pus cells and total sperm count normal sperm count (40-150 million) moderate (20-40 million), weak (1-20 million) and azoospermia (zero sperm or no sperm and classification according to viability in for orders of sperm count $/ \mathrm{ml}$ and found variable motility viscosity, volume/ml, total count, pus cells $/ \mathrm{ml}, \mathrm{RBCs} / \mathrm{ml}$, spermatogenic cells, abnormal forms from sample to other and this study represent that bacterial infection of the semen can have a direct role in spermatozoid parameters. Change and may result in men's infertility. In the present study 68 semens (Pur Culture)of bacterial infection were infected by different species bacteria but those infected by 
Evaluation of Escherichia coli infection control as the most

these bacteria it may be conducted that the bacteria causing genital tract infection can defect the morphology and the motility of men's spermatozoa.

Pathogenic Organisms Isolated From Seminal Fluid :( 68 pure Culture )

\section{Gram negative (rod-shape):}

31 isolates (45.6\%.) Escherichia coli, 3 isolates $(4.4 \%$.) Protus vuligar and 3 isolates (4.4\%)Pseudomonas aeruginosa

\section{Gram positive (cocci form):}

16 isolates $(23.5 \%)$ Streptococcus faecalis, 2 isolates $(2.9 \%)$ Streptococcus pyogenes, 10 isolates $(14.7 \%$.)Staphylococcus aureus and 3 isolates (4.4\%.)Staphylococcus saprophiticus.

Biochemical Identification criteria for Isolates Count .

(31)were found to be:grame -ve , Rod aerobes, Positive (+++) to Motility, Indol Production \& Ornithine decarboxylation), -ve to Urease, -ve to Oxidase: -ve, to Citrate utilization: $\mathrm{K} / \mathrm{A}$ to KIA (Kligler Iron Agar): K / A:

LIA(Lysine Iron Agar): K / K.

It is likely to be Escherichia coli

$\mathrm{A}=$ Acidic .

$\mathrm{K}=$ Alkaline $. \mathrm{H} 2 \mathrm{~S}=$ black .

Effect of E.Coli incubation periods on spermatozoa motility

Immediately after density gradient centrifugation, the sperm preparations had a sperm motility of $73.4 \%(n=30)$ (Graph 1) and with only $10.3 \%$ dead spermatozoa. Purified spermatozoa were incubated with E. coli, and sperm motility was studied using ordinary light microscopy. The spermatozoa started to clump immediately after mixing. After 5 min of incubation, small sperm agglutinations could be detected and, with time, the agglutinations increased in size. Repeating the experiments showed that the sizes and number of the agglutinates varied with 10-fold dilutions of E. coli and sperm obtained from different donors.

Graph 1: Percentage of motility of spermatozoa when incubated with live E. coli and control
After $1 \mathrm{~h}$ of incubation, for example, sperm motility has fallen to $57.1 \%$ and this was significantly different from the control incubation. Further completion of incubation at $6 \mathrm{~h}$ the percentage of motile spermatozoa left is $11 \%$ that is very low in comparison to control. Over the $6 \mathrm{~h}$ incubation period, these characteristics did not alter significantly in control population. Agglutination was determined for both the samples (Graph 2). In experimental group, the agglutination of bacteria was observed while in case of control no agglutination was observed $(\geq 5 \%$ ). At each time point, an aliquot of $3 \mu \mathrm{l}$ was mounted on slides, and after a few minutes the sperm stuck to the glass cover slips. A total of $3 \times 100$ spermatozoa were counted and the percentage of agglutination of sperm was determined.

Effect of E.coli incubation period on spermatozoa Agglutination

* Graph 2: Percentage agglutination of spermatozoa when incubated with live E. coli and control

\section{Experiment}

The Most Potent Antibiotics affecting E.coli : MBC(Minimal bactericidal concentration ) for these five antibiotics i.e. Amoxicillin, Ciprofloxacin, Erythromycin, Gentamicin and Penicillin $\mathrm{G}$ are shown in table 1 . These antimicrobial agents were used to inhibit the growth of E. coli and positive control was not treated with any antibiotic. High agglutination was observed in control. In experimental group, killed bacteria failed to bring about the same change

However : The bacterial growth was stopped by heating the $48 \mathrm{~h}$ old culture for $30 \mathrm{~min}$. Both of the culture (experiment $2 \& 3$ ) showed no growth on nutrient agar plates. Positive control was incubated at $37 \mathrm{oC}$. The heat treated $\mathrm{E}$. coli did not showed any changes in the sperm regarding to motility and viability. This shows the inference in motility is may be due to $\mathrm{E}$. coli and not the soluble factor. 
A.A Farrag et al .

Table (1): Full description of patients infected(265 )seminal fluid from (500sample )

\begin{tabular}{|c|c|c|c|c|c|c|c|c|c|}
\hline Semen EX.. Patient No.. & Age & $\begin{array}{l}\text { Vol. } \\
\text { /ml }\end{array}$ & $\begin{array}{l}\text { Viscosity } \\
\text { at } 30 \circ C\end{array}$ & $\begin{array}{l}\text { Total count } \\
/ \mathrm{ml}\end{array}$ & $\begin{array}{l}\text { Pus/ } \\
\text { H.P.F }\end{array}$ & $\begin{array}{l}\text { R.B.Cs } \\
\text { / H.P.F }\end{array}$ & $\begin{array}{c}\text { Spermatoge } \\
\text { nic.Cells } \\
/ \text { H.P.F }\end{array}$ & Viability & $\begin{array}{l}\text { Abn. } \\
\text { Sermp. } \\
\%\end{array}$ \\
\hline S & 21 & 6 & S. viscid & 78.000 .000 & $30-40$ & $2-3$ & $15-20$ & $\begin{array}{l}70 \% \text { A } \\
10 \% \text { SI. } \\
20 \% \text { D }\end{array}$ & $3-4$ \\
\hline 2 & 32 & 1 & V. viscid & 62.000 .000 & $10-15$ & $1-2$ & $6-8$ & $\begin{array}{l}40 \% \text { A } \\
20 \% \text { SI. } \\
40 \% \text { D }\end{array}$ & $6-8$ \\
\hline 3 & 22 & 3 & S. Viscid & 6.000 .000 & $50-60$ & $3-4$ & $15-20$ & $\begin{array}{l}40 \% \text { A. } \\
20 \% \text { S. } \\
40 \% \text { D. }\end{array}$ & $10-12$ \\
\hline 7 & 33 & 6 & L. viscid & 60.000 .000 & $15-20$ & $2-3$ & $1-2$ & $\begin{array}{l}70 \% \text { A. } \\
10 \% \text { S. } \\
20 \% \text { D. }\end{array}$ & $5-8$ \\
\hline 9 & 25 & 8 & S. viscid & 32.000 .000 & $\begin{array}{l}\text { Over } \\
100\end{array}$ & $2-3$ & $4-5$ & $\begin{array}{l}60 \% \text { A. } \\
10 \% \text { S. } \\
30 \% \text { D. }\end{array}$ & 10-12 \\
\hline 14 & 21 & 3 & S. viscid & 40.000 .000 & $\begin{array}{l}\text { Over } \\
100\end{array}$ & $3-4$ & $1-2$ & $\begin{array}{l}50 \% \text { A. } \\
20 \% \text { S. } \\
30 \% \text { D }\end{array}$ & 10-12 \\
\hline 15 & 30 & 4 & S. viscid & 40.000 .000 & $\begin{array}{l}\text { Over } \\
100\end{array}$ & $2-3$ & $1-2$ & $\begin{array}{l}45 \% \mathrm{~A} . \\
15 \% \mathrm{~S} . \\
40 \% \mathrm{D} .\end{array}$ & $15-20$ \\
\hline 27 & 27 & 3 & S. viscid & 38.000 .000 & $20-25$ & $2-3$ & $5-8$ & $\begin{array}{l}50 \% \text { A. } \\
20 \% \text { S. } \\
30 \% \text { D. }\end{array}$ & $12-15$ \\
\hline 28 & 31 & 3 & S. viscid & 3.000 .000 & $\begin{array}{l}\text { Over } \\
100\end{array}$ & $3-4$ & $6-7$ & $\begin{array}{l}40 \% \text { A. } \\
20 \% \text { S. } \\
40 \% \text { D. }\end{array}$ & $8-10$ \\
\hline 32 & 40 & 2 & S. viscid & 12.000 .000 & $10-15$ & $1-2$ & $2-3$ & $\begin{array}{l}30 \% \text { A. } \\
20 \% \text { S. } \\
50 \% \text { D. }\end{array}$ & $15-20$ \\
\hline 33 & 30 & 3 & S.Viscid & 40.000 .000 & $10-15$ & $1-2$ & $2-3$ & $\begin{array}{l}50 \% \text { A. } \\
20 \% \text { S. } \\
30 \% \text { D. }\end{array}$ & $12-15$ \\
\hline 38 & 22 & 1 & S. Viscid & 5.000 .000 & $15-20$ & $1-2$ & $1-2$ & $\begin{array}{l}50 \% \text { A. } \\
20 \% \text { S. } \\
30 \% \text { D. }\end{array}$ & $4-6$ \\
\hline 40 & 33 & 4 & S. viscid & 52.000 .000 & $50-60$ & $2-4$ & $2-3$ & $\begin{array}{l}60 \% \text { A. } \\
20 \% \text { S. } \\
20 \% \text { D. }\end{array}$ & $12-15$ \\
\hline 41 & 34 & 5 & S. viscid & 72.000 .000 & 10-12 & $1-2$ & $4-5$ & $\begin{array}{l}70 \% \text { A. } \\
15 \% \text { S. } \\
15 \% \text { D. }\end{array}$ & $6-8$ \\
\hline 46 & 25 & 3 & S. viscid & 85.000 .000 & $\begin{array}{l}\text { Over } \\
100\end{array}$ & $2-3$ & $4-6$ & $\begin{array}{l}60 \% \text { A. } \\
10 \% \text { S. } \\
30 \% \text { D. }\end{array}$ & $8-10$ \\
\hline 47 & 35 & 5 & S. Viscid & 60.000 .000 & $10-12$ & $1-2$ & $4-5$ & $\begin{array}{l}50 \% \text { A. } \\
10 \% \text { S. } \\
40 \% \text { D. }\end{array}$ & $8-10$ \\
\hline 49 & 40 & 3 & S. viscid & 70.000 .000 & $\begin{array}{l}\text { Over } \\
100\end{array}$ & $2-3$ & $4-6$ & $\begin{array}{l}75 \% \text { A. } \\
15 \% \text { S. } \\
10 \% \text { D. }\end{array}$ & $6-8$ \\
\hline 50 & 23 & 5 & S. viscid & 76.000 .000 & $20-25$ & $2-4$ & $8-10$ & $\begin{array}{l}\text { A. \%65 } \\
15 \% \text { S. } \\
20 \% \text { D. }\end{array}$ & $8-10$ \\
\hline 54 & 23 & 1 & S. viscid & 6.000 .000 & $10-15$ & $2-4$ & $2-3$ & $\begin{array}{l}40 \% \text { A. } \\
20 \% \text { S. } \\
40 \% \text { D. }\end{array}$ & $15-20$ \\
\hline 55 & 30 & 5 & S. viscid & 82.000 .000 & $20-25$ & $3-5$ & $1-2$ & $\begin{array}{l}80 \% \text { A. } \\
10 \% \text { S. } \\
10 \% \text { D. }\end{array}$ & $4-5$ \\
\hline 60 & 40 & 5 & S. viscid & 32.000 .000 & $30-35$ & $2-4$ & $15-20$ & $\begin{array}{l}50 \% \text { A. } \\
20 \% \text { S. } \\
30 \% \text { D. }\end{array}$ & $8-10$ \\
\hline 67 & 26 & 10 & S. viscid & 16.000 .000 & $15-20$ & $2-4$ & $4-6$ & $\begin{array}{l}40 \% \text { A. } \\
20 \% \text { S. } \\
40 \% \text { D. }\end{array}$ & $10-12$ \\
\hline 71 & 30 & 4 & S. Viscid & 5.000 .000 & $20-25$ & $2-3$ & $10-12$ & $\begin{array}{l}40 \% \text { A. } \\
20 \% \text { S. } \\
40 \% \text { D }\end{array}$ & $12-15$ \\
\hline 72 & 22 & 5 & S. Viscid & 72.000 .000 & $50-60$ & $2-4$ & $2-3$ & $\begin{array}{l}60 \% \text { A. } \\
10 \% \text { S. } \\
30 \% \text { D. }\end{array}$ & $6-8$ \\
\hline 85 & 23 & 6 & S. viscid & 62.000 .000 & $10-15$ & $2-3$ & $2-3$ & $\begin{array}{l}30 \% \text { A. } \\
10 \% \text { S. } \\
60 \% \text { D. }\end{array}$ & $8-10$ \\
\hline 86 & 57 & 4 & S. viscid & 76.000 .000 & $7-8$ & $1-2$ & $4-5$ & $\begin{array}{l}80 \% \text { A. } \\
10 \% \text { S. } \\
10 \% \text { D. }\end{array}$ & $4-6$ \\
\hline 89 & 35 & 4 & S. Viscid & 60.000 .000 & $\begin{array}{l}\text { Over } \\
100\end{array}$ & $2-3$ & $2-3$ & $\begin{array}{l}60 \% \mathrm{~A} . \\
10 \% \mathrm{~S} \\
30 \% \mathrm{D}\end{array}$ & $12-14$ \\
\hline 91 & 32 & 5 & S. viscid & 56.000 .000 & $\begin{array}{l}\text { Over } \\
100\end{array}$ & $3-4$ & $10-15$ & $\begin{array}{l}60 \% \text { A. } \\
20 \% \text { S. } \\
20 \% \text { D. }\end{array}$ & $4-5$ \\
\hline 92 & 35 & 3 & S. viscid & 62.000 .000 & 15- 20 & $2-3$ & $8-10$ & $\begin{array}{l}60 \% \text { A. } \\
10 \% \text { S. } \\
30 \% \text { D. }\end{array}$ & $8-10$ \\
\hline 96 & 22 & 2 & S. viscid & 50.000 .000 & $40-50$ & $2-3$ & $15-20$ & $\begin{array}{l}60 \% \text { A. } \\
20 \% \text { S. } \\
20 \% \text { D. }\end{array}$ & $4-5$ \\
\hline 98 & 26 & 3 & S. Viscid & 52.000 .000 & $\begin{array}{l}\text { Over } \\
100\end{array}$ & $2-3$ & $10-15$ & $\begin{array}{l}65 \% \text { A } \\
15 \% \text { S. } \\
20 \% \text { D }\end{array}$ & $5-7$ \\
\hline 100 & 36 & 3 & V. viscid & 76.000 .000 & $10-15$ & $1-2$ & $3-4$ & $\begin{array}{l}70 \% \text { A. } \\
10 \% \text { S. } \\
20 \% \text { D. }\end{array}$ & 4-6 \\
\hline 104 & 25 & 2 & S. viscid & 8.400 .000 & $\begin{array}{l}\text { Over } \\
100\end{array}$ & $3-4$ & $4-6$ & $\begin{array}{l}40 \% \text { A. } \\
20 \% \text { S. } \\
40 \% \text { D. }\end{array}$ & $10-12$ \\
\hline 107 & 25 & 2 & S. Viscid & 60.000 .000 & $\begin{array}{l}\text { Over } \\
100\end{array}$ & $3-4$ & $8-10$ & $\begin{array}{l}60 \% \text { A. } \\
20 \% \text { S. } \\
20 \% \text { D. }\end{array}$ & $6-8$ \\
\hline 128 & 27 & 4 & S. viscid & 75.000 .000 & $15-20$ & $3-5$ & $3-4$ & $\begin{array}{l}78 \% \text { A. } \\
10 \% \mathrm{~S} . \\
12 \% \mathrm{D} .\end{array}$ & $4-5$ \\
\hline 129 & 20 & 6 & S. Viscid & 75.000 .000 & $40-50$ & $3-4$ & $3-4$ & $\begin{array}{l}70 \% \text { A. } \\
10 \% \text { S. } \\
20 \% \text { D. }\end{array}$ & $8-10$ \\
\hline 135 & 28 & 3 & S. Viscid & 20.000 .000 & $10-15$ & $0-1$ & $2-4$ & $\begin{array}{l}55 \% \text { A. } \\
20 \% \text { S. } \\
25 \% \text { D. }\end{array}$ & $12-15$ \\
\hline 145 & 24 & 1 & S. viscid & 21.400 .000 & $10-15$ & $0-1$ & $8-10$ & $\begin{array}{l}20 \% \text { A. } \\
10 \% \text { S. } \\
70 \% \text { D. }\end{array}$ & $12-15$ \\
\hline
\end{tabular}


Evaluation of Escherichia coli infection control as the most

\begin{tabular}{|c|c|c|c|c|c|c|c|c|c|}
\hline 156 & 29 & 5 & S. Viscid & 74.000 .000 & $10-15$ & $2-3$ & $6-8$ & $\begin{array}{l}70 \% \mathrm{~A} . \\
10 \% \mathrm{~S} . \\
20 \% \mathrm{D}\end{array}$ & $5-6$ \\
\hline 157 & 24 & 5 & S. viscid & 70.000 .000 & $20-25$ & $3-4$ & $6-8$ & $\begin{array}{l}60 \% \mathrm{~A} . \\
10 \% \mathrm{~S} . \\
30 \% \mathrm{D} .\end{array}$ & $10-12$ \\
\hline 158 & 19 & 4 & S. viscid & 64.000 .000 & $10-15$ & $0-1$ & $4-5$ & $\begin{array}{l}70 \% \mathrm{~A} . \\
10 \% \mathrm{~S} . \\
20 \% \mathrm{D} .\end{array}$ & $5-6$ \\
\hline 159 & 21 & 5 & S. viscid & 65.000 .000 & $10-15$ & $0-1$ & $8-10$ & $\begin{array}{l}60 \% \text { A. } \\
10 \% \text { S. } \\
30 \% \text { D. }\end{array}$ & $10-12$ \\
\hline 160 & 26 & 5 & S. viscid & 74.000 .000 & $10-15$ & $0-1$ & $6-8$ & $\begin{array}{l}60 \% \mathrm{~A} . \\
10 \% \mathrm{~S} . \\
30 \% \mathrm{D} .\end{array}$ & $6-8$ \\
\hline 162 & 23 & 5 & S. Viscid & 94.000 .000 & $10-15$ & $0-1$ & $6-8$ & $\begin{array}{l}80 \% \mathrm{~A} . \\
10 \% \mathrm{~S} . \\
10 \% \mathrm{D} .\end{array}$ & $6-8$ \\
\hline 165 & 26 & 3 & S. Viscid & 18.000 .000 & $20-25$ & $2-3$ & $2-3$ & $\begin{array}{l}50 \% \mathrm{~A} . \\
20 \% \mathrm{~S} . \\
20 \% \mathrm{D} .\end{array}$ & $4-5$ \\
\hline 168 & 31 & 5 & S. viscid & 48.000 .000 & $8-10$ & $0-1$ & $2-3$ & $\begin{array}{c}70 \% \text { A. } \\
5 \% \text { S. } \\
25 \% \text { D. }\end{array}$ & $4-5$ \\
\hline 169 & 26 & 4 & S. Viscid & 16.000 .000 & $15-20$ & $2-3$ & $4-5$ & $\begin{array}{l}40 \% \mathrm{~A} . \\
20 \% \mathrm{~S} . \\
40 \% \mathrm{D} .\end{array}$ & $5-6$ \\
\hline 171 & 45 & 4 & L. Viscid & 10.200 .000 & $10-12$ & $2-3$ & $15-20$ & $\begin{array}{l}40 \% \text { A. } \\
20 \% \text { S. } \\
40 \% \text { D. }\end{array}$ & $6-8$ \\
\hline 183 & 20 & 2 & S. Viscid & 6.000 .000 & $50-60$ & $2-3$ & $10-12$ & $\begin{array}{l}40 \% \text { A. } \\
20 \% \text { S. } \\
40 \% \text { D. }\end{array}$ & $5-6$ \\
\hline 208 & 23 & 4.0 & S.Viscid & 65.000 .000 & $\begin{array}{c}\text { Over } \\
100\end{array}$ & $1-2$ & $3-5$ & $\begin{array}{l}60 \% \text { A. } \\
30 \% \text { S. } \\
10 \% \text { D. }\end{array}$ & $8-10$ \\
\hline 213 & 27 & 2.0 & S. viscid & 62.000 .000 & $10-12$ & $1-2$ & $5-6$ & $\begin{array}{l}60 \% \text { A. } \\
20 \% \text { S. } \\
20 \% \text { D. }\end{array}$ & $3-4$ \\
\hline 225 & 30 & 4.0 & S.viscid. & 66.000 .000 & $15-20$ & $2-3$ & $2-3$ & $\begin{array}{l}60 \% \mathrm{~A} . \\
10 \% \mathrm{~S} . \\
30 \% \mathrm{D} .\end{array}$ & $10-12$ \\
\hline 234 & 33 & 2.5 & S. viscid & 44.000 .000 & $20-25$ & $2-3$ & $4-5$ & $\begin{array}{l}60 \% \text { A. } \\
20 \% \text { S. } \\
20 \% \text { D. }\end{array}$ & $10-12$ \\
\hline 244 & 23 & 3 & S. Viscid & 30.200 .000 & $10-12$ & $1-2$ & $8-10$ & $\begin{array}{l}40 \% \text { A. } \\
20 \% \mathrm{~S} . \\
40 \% \mathrm{D} .\end{array}$ & $10-12$ \\
\hline 247 & 25 & 5 & S. viscid & 60.000 .000 & $50-60$ & $2-3$ & $4-6$ & $\begin{array}{l}75 \% \mathrm{~A} . \\
10 \% \mathrm{~S} . \\
15 \% \mathrm{D} .\end{array}$ & $10-12$ \\
\hline 253 & 32 & 3 & S. Viscid & 40.000 .000 & $15-20$ & $1-2$ & $15-20$ & $\begin{array}{l}40 \% \mathrm{~A} . \\
20 \% \mathrm{~S} . \\
40 \% \mathrm{D} .\end{array}$ & $12-15$ \\
\hline 255 & 21 & 5 & S. viscid & 10.000 .000 & $10-15$ & $0-1$ & $20-30$ & $\begin{array}{l}40 \% \text { A. } \\
20 \% \text { S. } \\
40 \% \text { D. }\end{array}$ & $10-12$ \\
\hline 256 & 30 & 5 & S. viscid & 3.000 .000 & Over100 & $4-5$ & $5-8$ & $\begin{array}{l}50 \% \mathrm{~A} . \\
20 \% \mathrm{~S} . \\
30 \% \mathrm{D} .\end{array}$ & $8-10$ \\
\hline 257 & 37 & 4 & S. Viscid & 6.000 .000 & $20-25$ & $0-1$ & $1-2$ & $\begin{array}{l}40 \% \text { A. } \\
25 \% \text { S. } \\
35 \% \text { D. }\end{array}$ & $12-15$ \\
\hline 258 & 40 & 3 & S. viscid & 1.000 .000 & $15-20$ & $2-3$ & $1-2$ & $\begin{array}{l}30 \% \mathrm{~A} . \\
20 \% \mathrm{~S} . \\
50 \% \mathrm{D} .\end{array}$ & $12-15$ \\
\hline 259 & 25 & 4 & S. viscid & 18.000 .000 & $6-8$ & $0-1$ & $5-6$ & $\begin{array}{l}60 \% \text { A } \\
20 \% \text { S. } \\
20 \% \text { D. }\end{array}$ & $8-10$ \\
\hline 260 & 30 & 5 & S. viscid & Asospermia & $20-25$ & $1-2$ & Zero & Zero & Zero \\
\hline 261 & 22 & 6 & S. viscid & Asospermia & $10-15$ & $0-1$ & $0-1$ & Zero. & Zero \\
\hline 262 & 29 & 5 & S. Viscid & 64.000 .000 & $10-12$ & $0-1$ & $1-2$ & $\begin{array}{l}70 \% \mathrm{~A} . \\
10 \% \mathrm{~S} . \\
20 \% \mathrm{D} .\end{array}$ & $10-12$ \\
\hline 263 & 26 & 3 & S. Viscid & 16.000 .000 & $8-10$ & $1-2$ & $2-3$ & $\begin{array}{l}60 \% \mathrm{~A} . \\
10 \% \mathrm{~S} . \\
30 \% \mathrm{D} .\end{array}$ & $8-10$ \\
\hline 264 & 32 & 3 & S. Viscid & 1.000 .000 & $25-30$ & $1-2$ & $2-3$ & Zero & $10-12$ \\
\hline 265 & 37 & 5 & S. Viscid & 74.000 .000 & $\begin{array}{c}\text { Over } \\
100\end{array}$ & $1-2$ & $3-5$ & $\begin{array}{l}70 \% \mathrm{~A} . \\
10 \% \mathrm{~S} . \\
20 \% \mathrm{D} .\end{array}$ & $5-6$ \\
\hline
\end{tabular}

D: Dead (immotile) S. :Semi , A. : Active , S. : Sluggish, V.: Very : L. : Low 
Table (2):Sensitivity Test of the Infected(68) Semen Fluid tested isolates From (265 Seminal Fluid )against different antibiotics :

\begin{tabular}{|c|c|c|c|c|c|c|c|c|c|c|c|c|}
\hline \multirow{2}{*}{$\begin{array}{l}\text { Sample } \\
\text { Number }\end{array}$} & \\
\hline & CIP & AK & OFX & GM & AMP & $\mathrm{DA}$ & $E$ & $P$ & CRO & SAM & $\mathrm{FL}$ & AZM \\
\hline 1 & ++++ & ++ & +++ & ++ & + & $\mathbf{R}$ & $\mathbf{R}$ & $\mathbf{R}$ & $\mathbf{R}$ & $\mathbf{R}$ & $\mathbf{R}$ & $\mathbf{R}$ \\
\hline 2 & +++ & +++ & ++ & $\mathbf{R}$ & + & $\mathbf{R}$ & $\mathbf{R}$ & $\mathbf{R}$ & $\mathbf{R}$ & $\mathbf{R}$ & $\mathbf{R}$ & $\mathbf{R}$ \\
\hline 3 & +++ & +++ & ++ & $\mathbf{R}$ & + & $R$ & $\mathbf{R}$ & $\mathbf{R}$ & $\mathbf{R}$ & $\mathbf{R}$ & $\mathbf{R}$ & $R$ \\
\hline 7 & +++ & $\mathrm{R}$ & +++ & + & $R$ & $\mathrm{R}$ & ++ & $R$ & $R$ & $R$ & $\mathrm{R}$ & $\mathrm{R}$ \\
\hline 9 & ++ & + & $\begin{array}{l}R \\
\end{array}$ & $\begin{array}{l}R \\
\end{array}$ & $R$ & +++ & + & $R$ & $R$ & $R$ & +++ & $\begin{array}{l}R \\
\end{array}$ \\
\hline 14 & +++ & +++ & $R$ & $R$ & $\mathrm{R}$ & R & ++ & + & $\mathrm{R}$ & $R$ & 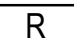 & $R$ \\
\hline 15 & +++ & $\mathrm{R}$ & $R$ & $R$ & $\mathrm{R}$ & $\mathrm{R}$ & ++ & ++ & $\mathrm{R}$ & $\mathrm{R}$ & $\mathrm{R}$ & + \\
\hline 27 & +++ & ++ & $R$ & $R$ & + & $R$ & $R$ & $R$ & $\mathrm{R}$ & $\mathrm{R}$ & $R$ & $\mathrm{R}$ \\
\hline 28 & +++ & $\mathrm{R}$ & $R$ & $R$ & $R$ & $R$ & $R$ & $R$ & $R$ & $\mathrm{R}$ & $R$ & ++ \\
\hline 32 & +++ & $\mathrm{R}$ & +++ & $R$ & $\bar{R}$ & $\bar{R}$ & $R$ & $R$ & + & ++ & $\bar{R}$ & $R$ \\
\hline 32 & ++ & $\mathrm{R}$ & +++ & $\mathrm{R}$ & $\mathrm{R}$ & $\mathrm{R}$ & $R$ & $R$ & + & ++ & $R$ & $R$ \\
\hline 33 & +++ & ++ & + & $\begin{array}{l}R \\
\end{array}$ & $R$ & $R$ & $R$ & $R$ & $R$ & $\mathrm{R}$ & $\mathrm{R}$ & $\mathrm{R}$ \\
\hline 38 & +++ & $R$ & $\mathrm{R}$ & $R$ & $\mathrm{R}$ & $\mathrm{R}$ & ++ & $\mathrm{R}$ & $R$ & $\mathrm{R}$ & $\mathrm{R}$ & +++ \\
\hline 40 & 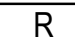 & $\bar{R}$ & + & 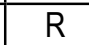 & $R$ & $\mathrm{R}$ & $\mathrm{R}$ & $\mathrm{R}$ & +++ & $\mathrm{R}$ & $R$ & ++ \\
\hline 41 & $R$ & ++ & $R$ & + & $R$ & +++ & $R$ & $R$ & $\mathrm{R}$ & $R$ & $R$ & $R$ \\
\hline 45 & +++ & ++ & $R$ & $R$ & $R$ & 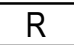 & + & $R$ & $R$ & $R$ & $R$ & $R$ \\
\hline 46 & +++ & ++ & $\begin{array}{l}R \\
\end{array}$ & $\begin{array}{l}R \\
\end{array}$ & $\mathrm{R}$ & +++ & + & $R$ & $\mathrm{R}$ & $R$ & $\mathrm{R}$ & $\begin{array}{l}R \\
\end{array}$ \\
\hline 47 & + & + & $R$ & $R$ & +++ & ++ & ++ & $R$ & $R$ & $R$ & $\mathrm{R}$ & $R$ \\
\hline 49 & +++ & ++ & $R$ & $R$ & $R$ & $R$ & + & $R$ & $R$ & $R$ & $R$ & $R$ \\
\hline 50 & ++ & $R$ & $R$ & $R$ & $R$ & $R$ & $\mathrm{R}$ & $R$ & +++ & $R$ & $R$ & $R$ \\
\hline 54 & $\mathrm{R}$ & + & +++ & $\mathrm{R}$ & $\mathrm{R}$ & $\mathrm{R}$ & $\mathrm{R}$ & $\mathrm{R}$ & $\mathrm{R}$ & ++ & +++ & $\mathrm{R}$ \\
\hline 55 & ++ & ++ & $\mathrm{R}$ & $R$ & $\mathrm{R}$ & $\mathrm{R}$ & +++ & $\mathrm{R}$ & $\mathrm{R}$ & $\mathrm{R}$ & $\mathrm{R}$ & $R$ \\
\hline 60 & +++ & +++ & $\mathrm{R}$ & ++ & + & $\mathrm{R}$ & + & $\mathrm{R}$ & $\mathrm{R}$ & $\mathrm{R}$ & $\mathrm{R}$ & $\mathrm{R}$ \\
\hline 67 & ++ & +++ & $\mathrm{R}$ & $\mathrm{R}$ & $\mathrm{R}$ & $\mathrm{R}$ & ++ & $\mathrm{R}$ & $\mathrm{R}$ & $\mathrm{R}$ & $\mathrm{R}$ & $\mathrm{R}$ \\
\hline 71 & +++ & + & $\bar{R}$ & 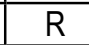 & $R$ & $\mathrm{R}$ & ++ & $\mathrm{R}$ & $\mathrm{R}$ & $\mathrm{R}$ & $\mathrm{R}$ & 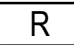 \\
\hline 72 & +++ & ++ & $R$ & $R$ & $\mathrm{R}$ & $R$ & + & $R$ & $\mathrm{R}$ & ++ & $\mathrm{R}$ & $R$ \\
\hline 85 & +++ & $R$ & $R$ & +++ & ++ & $R$ & $R$ & $R$ & $R$ & + & $R$ & $R$ \\
\hline 86 & +++ & $R$ & + & R & $\mathrm{R}$ & $R$ & ++ & $\mathrm{R}$ & $\mathrm{R}$ & $R$ & $\mathrm{R}$ & $R$ \\
\hline 89 & +++ & $\mathrm{R}$ & $\mathrm{R}$ & ++ & + & $R$ & ++ & $R$ & $\mathrm{R}$ & $\mathrm{R}$ & $\mathrm{R}$ & $R$ \\
\hline 91 & +++ & $R$ & + & $\mathrm{R}$ & $R$ & $R$ & $R$ & $R$ & $R$ & ++ & $R$ & $R$ \\
\hline 92 & +++ & $\mathrm{R}$ & $\mathrm{R}$ & ++ & +++ & + & $\mathrm{R}$ & $\mathrm{R}$ & + & $\mathrm{R}$ & $\mathrm{R}$ & $\mathrm{R}$ \\
\hline 96 & +++ & $R$ & ++ & R & $\mathrm{R}$ & $R$ & + & $R$ & $R$ & $R$ & $R$ & $\mathrm{R}$ \\
\hline 98 & +++ & $R$ & $\mathrm{R}$ & +++ & $R$ & $R$ & $R$ & $R$ & ++ & + & $R$ & $R$ \\
\hline 100 & +++ & ++ & $\mathrm{R}$ & $\mathrm{R}$ & $\mathrm{R}$ & $\mathrm{R}$ & $\mathrm{R}$ & $\mathrm{R}$ & $\mathrm{R}$ & + & $\mathrm{R}$ & $\mathrm{R}$ \\
\hline 104 & + & +++ & $R$ & ++ & $R$ & $R$ & ++ & $R$ & $R$ & $R$ & $R$ & $R$ \\
\hline 107 & +++ & $\mathrm{R}$ & +++ & $\mathrm{R}$ & $R$ & $\mathrm{R}$ & ++ & $R$ & $R$ & $R$ & $R$ & $R$ \\
\hline 128 & +++ & ++ & $\mathrm{R}$ & $\mathrm{R}$ & $\mathrm{R}$ & $\mathrm{R}$ & $\mathrm{R}$ & $\mathrm{R}$ & $\mathrm{R}$ & $\mathrm{R}$ & $\mathrm{R}$ & $R$ \\
\hline 129 & +++ & $\mathrm{R}$ & +++ & $\mathrm{R}$ & $R$ & $R$ & $\mathrm{R}$ & $R$ & ++ & + & $\mathrm{R}$ & $\mathrm{R}$ \\
\hline 135 & $\mathrm{R}$ & $R$ & +++ & $R$ & ++ & $R$ & $R$ & $\mathrm{R}$ & $\mathrm{R}$ & $R$ & ++ & $\mathrm{R}$ \\
\hline 145 & $\mathrm{R}$ & $R$ & +++ & $R$ & $\mathrm{R}$ & $\mathrm{R}$ & $\mathrm{R}$ & $\mathrm{R}$ & ++ & $R$ & $\mathrm{R}$ & $\mathrm{R}$ \\
\hline 156 & +++ & $R$ & ++ & $\mathrm{R}$ & + & $R$ & $\mathrm{R}$ & $R$ & ++ & $R$ & $\mathrm{R}$ & $\mathrm{R}$ \\
\hline 157 & +++ & $R$ & ++ & $\bar{R}$ & $R$ & $\mathrm{R}$ & + & $R$ & $\mathrm{R}$ & $\mathrm{R}$ & $\mathrm{R}$ & $\mathrm{R}$ \\
\hline
\end{tabular}


Table ( 2 ): Cont.

\begin{tabular}{|c|c|c|c|c|c|c|c|c|c|c|c|c|}
\hline $\begin{array}{l}\text { Sample } \\
\text { number }\end{array}$ & & & OFX & \multicolumn{2}{|c|}{\begin{tabular}{|l|l|} 
GM & AMP \\
\end{tabular}} & $\mathrm{DA}$ & $E$ & $\mathbf{P}$ & CRO & SAM & $\mathrm{FL}$ & AZM \\
\hline 158 & ++ & $\mathrm{R}$ & +++ & $\mathrm{R}$ & + & $\mathrm{R}$ & $\mathrm{R}$ & $\mathrm{R}$ & $\mathrm{R}$ & +++ & $\mathrm{R}$ & $\mathrm{R}$ \\
\hline 159 & +++ & $\mathrm{R}$ & ++ & $\mathrm{R}$ & $\mathrm{R}$ & $\mathrm{R}$ & $\mathrm{R}$ & $\mathrm{R}$ & + & $\mathrm{R}$ & $\mathrm{R}$ & $\mathrm{R}$ \\
\hline 160 & $\mathrm{R}$ & ++ & +++ & $\mathrm{R}$ & $\mathrm{R}$ & $\mathrm{R}$ & $\mathrm{R}$ & $\mathrm{R}$ & +++ & $\mathrm{R}$ & $\mathrm{R}$ & $\mathrm{R}$ \\
\hline 162 & +++ & ++ & $\mathrm{R}$ & $\mathrm{R}$ & $\mathrm{R}$ & $\mathrm{R}$ & $\mathrm{R}$ & $\mathrm{R}$ & $\mathrm{R}$ & $\mathrm{R}$ & $\mathrm{R}$ & ++ \\
\hline 165 & +++ & $\mathrm{R}$ & ++ & $\mathrm{R}$ & $\mathrm{R}$ & $\mathrm{R}$ & $\bar{R}$ & $\bar{R}$ & $\mathrm{R}$ & $\mathrm{R}$ & $\bar{R}$ & $\bar{R}$ \\
\hline 168 & +++ & $\mathrm{R}$ & +++ & $\mathrm{R}$ & + & $\mathrm{R}$ & $\mathrm{R}$ & $\mathrm{R}$ & + & ++ & $\mathrm{R}$ & $\mathrm{R}$ \\
\hline 169 & +++ & ++ & $\mathrm{R}$ & $\mathrm{R}$ & $\mathrm{R}$ & $\mathrm{R}$ & $\mathrm{R}$ & $\mathrm{R}$ & +++ & $\mathrm{R}$ & + & $\mathrm{R}$ \\
\hline 171 & ++ & $\mathrm{R}$ & ++ & $\mathrm{R}$ & +++ & $\mathrm{R}$ & $\mathrm{R}$ & $\mathrm{R}$ & ++ & $\mathrm{R}$ & $\mathrm{R}$ & $\mathrm{R}$ \\
\hline 172 & +++ & ++ & $\mathrm{R}$ & + & $\mathrm{R}$ & $\mathrm{R}$ & $\mathrm{R}$ & $\mathrm{R}$ & +++ & $\mathrm{R}$ & $\mathrm{R}$ & $\mathrm{R}$ \\
\hline 183 & +++ & ++ & ++ & ++ & $R$ & $\mathrm{R}$ & $\mathrm{R}$ & $\mathrm{R}$ & $\mathrm{R}$ & $R$ & $R$ & $R$ \\
\hline 208 & +++ & + & $\mathrm{R}$ & $\mathrm{R}$ & $\mathrm{R}$ & $\mathrm{R}$ & +++ & $\mathrm{R}$ & $\mathrm{R}$ & $\mathrm{R}$ & $\mathrm{R}$ & $\mathrm{R}$ \\
\hline 213 & +++ & $\mathrm{R}$ & +++ & $\mathrm{R}$ & + & $\mathrm{R}$ & $\mathrm{R}$ & $\mathrm{R}$ & $\mathrm{R}$ & ++ & $\bar{R}$ & $\bar{R}$ \\
\hline 225 & $\mathrm{R}$ & ++ & $\mathrm{R}$ & + & $\mathrm{R}$ & +++ & $\mathrm{R}$ & $\mathrm{R}$ & $\mathrm{R}$ & $\mathrm{R}$ & $\mathrm{R}$ & $\mathrm{R}$ \\
\hline 234 & +++ & $\mathrm{R}$ & $\mathrm{R}$ & ++ & + & $\mathrm{R}$ & $\mathrm{R}$ & $\mathrm{R}$ & $\mathrm{R}$ & $\mathrm{R}$ & $R$ & $\mathrm{R}$ \\
\hline 244 & +++ & ++ & $\mathrm{R}$ & $\mathrm{R}$ & $\mathrm{R}$ & $\mathrm{R}$ & $\mathrm{R}$ & $\mathrm{R}$ & $\mathrm{R}$ & + & $\mathrm{R}$ & $\mathrm{R}$ \\
\hline 247 & +++ & +++ & $\mathrm{R}$ & $\mathrm{R}$ & $\mathrm{R}$ & $\mathrm{R}$ & $\mathrm{R}$ & + & ++ & ++ & +++ & $\mathrm{R}$ \\
\hline 253 & +++ & ++ & $\mathrm{R}$ & $\mathrm{R}$ & $\mathrm{R}$ & $\mathrm{R}$ & + & $\mathrm{R}$ & $\mathrm{R}$ & $\mathrm{R}$ & $\mathrm{R}$ & $\mathrm{R}$ \\
\hline 255 & +++ & +++ & $\mathrm{R}$ & $\mathrm{R}$ & $\mathrm{R}$ & $\mathrm{R}$ & ++ & $\mathrm{R}$ & $\mathrm{R}$ & $\mathrm{R}$ & $\mathrm{R}$ & $\mathrm{R}$ \\
\hline 256 & + & ++ & $\mathrm{R}$ & $\mathrm{R}$ & ++ & +++ & $\mathrm{R}$ & + & $\mathrm{R}$ & $\mathrm{R}$ & $\mathrm{R}$ & + \\
\hline 257 & +++ & $\bar{R}$ & $\mathrm{R}$ & $\mathrm{R}$ & +++ & ++ & $\mathrm{R}$ & $\mathrm{R}$ & $\mathrm{R}$ & $\mathrm{R}$ & $\bar{R}$ & ++ \\
\hline 258 & ++ & $\mathrm{R}$ & $\mathrm{R}$ & $\mathrm{R}$ & ++ & $\mathrm{R}$ & ++ & $\mathrm{R}$ & $\mathrm{R}$ & $\mathrm{R}$ & $\mathrm{R}$ & + \\
\hline 259 & +++ & $\mathrm{R}$ & $\mathrm{R}$ & $\mathrm{R}$ & ++ & $\mathrm{R}$ & $\mathrm{R}$ & $\mathrm{R}$ & $\mathrm{R}$ & $\mathrm{R}$ & $\bar{R}$ & +++ \\
\hline 260 & +++ & ++ & $\mathrm{R}$ & $\mathrm{R}$ & $\mathrm{R}$ & $\mathrm{R}$ & $\mathrm{R}$ & $\mathrm{R}$ & $\mathrm{R}$ & $\mathrm{R}$ & + & $\mathrm{R}$ \\
\hline 261 & +++ & $\mathrm{R}$ & $\mathrm{R}$ & $\mathrm{R}$ & ++ & $\mathrm{R}$ & $\mathrm{R}$ & $\mathrm{R}$ & $\mathrm{R}$ & $\mathrm{R}$ & R & +++ \\
\hline 262 & +++ & ++ & $\mathrm{R}$ & $\mathrm{R}$ & $\mathrm{R}$ & $\mathrm{R}$ & $\mathrm{R}$ & $\mathrm{R}$ & $\mathrm{R}$ & ++ & $R$ & $\mathrm{R}$ \\
\hline 263 & +++ & ++ & +++ & $\mathrm{R}$ & $\mathrm{R}$ & + & $\mathrm{R}$ & $\mathrm{R}$ & $\mathrm{R}$ & $\mathrm{R}$ & $R$ & $\mathrm{R}$ \\
\hline 264 & +++ & ++ & $\mathrm{R}$ & +++ & ++ & $\mathrm{R}$ & + & $\mathrm{R}$ & $\mathrm{R}$ & $\mathrm{R}$ & $R$ & $\mathrm{R}$ \\
\hline 265 & +++ & $\mathrm{R}$ & $\mathrm{R}$ & $\mathrm{R}$ & + & $\mathrm{R}$ & $\mathrm{R}$ & $\mathrm{R}$ & $\mathrm{R}$ & ++ & $\mathrm{R}$ & ++ \\
\hline $\begin{array}{l}\text { * CIP } \\
* \mathbf{A K}= \\
* \mathbf{O F X} \\
* \mathbf{G M} \\
* \mathbf{A M} \\
* \mathbf{D A} \\
* \mathbf{R}= \\
* \text { Very }\end{array}$ & $\begin{array}{l}\text { Cipro } \\
\text { Amiki } \\
=\text { Oflo } \\
=\text { Gran } \\
=\text { Am } \\
\text { Clidar } \\
\text { esistar } \\
\text { Sensiti }\end{array}$ & $\begin{array}{l}\text { in } \\
\text { xacin } \\
\text { ycin } \\
\text { icillin } \\
\text { nycin } \\
\text { t }\end{array}$ & & & & $\begin{array}{l}\mathbf{E}= \\
* \mathbf{P}= \\
* \mathbf{C R} \\
* \mathbf{S A} \\
* \mathbf{F L} \\
* \mathbf{A Z} \\
* \mathbf{L E}\end{array}$ & $\begin{array}{l}=\text { Ery } \\
=\text { peni } \\
\text { O = } \\
M= \\
=\text { Fl } \\
M= \\
\text { ss Se }\end{array}$ & $\begin{array}{l}\text { irom } \\
\text { illin } \\
\text { eftria } \\
\text { nasy } \\
\text { mox } \\
\text { zith }\end{array}$ & $\begin{array}{l}\text { ycin } \\
\text { xone }\end{array}$ & & & \\
\hline
\end{tabular}


Table (3) : Pus cells : Classificationaccording to sperm count/ml Through Five orders of pus cells Prevalence From ( 265 Seminal Fluid )

\begin{tabular}{||c|c|c|c|c|c||}
\hline \multicolumn{1}{|c|}{ Pus Cells /H.P.F. } & $\mathbf{0 - 5}$ & $\mathbf{1 0 - 2 0}$ & $\mathbf{2 0 - 5 0}$ & Over $\mathbf{5 0}$ & Over $\mathbf{1 0 0}$ \\
\hline Total= & 60 & 157 & 19 & 9 & 20 \\
\hline $\begin{array}{l}\text { Normospermia (Normal) } \\
\mathbf{4 0 - 1 5 0} \text { million) }\end{array}$ & 20 & 93 & 13 & 5 & 15 \\
\hline $\begin{array}{l}\text { (Normospermia } \\
\text { (Moderate) =26 } \\
(\mathbf{2 0 - 4 0} \text { million) }\end{array}$ & 10 & 10 & 3 & 2 & 1 \\
\hline $\begin{array}{l}\text { Olegospermia (Weak ) } \\
(\mathbf{1}-\mathbf{2 0} \text { million) }\end{array}$ & 16 & 51 & 2 & 1 & 3 \\
\hline $\mathbf{2 0}=$ Asospermia ( no sperm) & 14 & 3 & 1 & 1 & 1 \\
\hline
\end{tabular}

Table( 4 ): Classification of Sperm count cases according toViability in four Orders of sperm count/ $\mathrm{ml}$ ( 265 Sample)

\begin{tabular}{||l|c|c|c||}
\hline \hline Viability\% & $\begin{array}{c}\text { Active = 167 } \\
(50-100 \%)\end{array}$ & $\begin{array}{c}\text { Sluggish = 48 } \\
(10-50 \%)\end{array}$ & Dead = 30 \\
\hline $\begin{array}{l}\text { Normospermia (Normal) } \\
(40-150 \text { million ) } 146\end{array}$ & 110 & 20 & 16 \\
\hline $\begin{array}{l}\text { Normospermia (Moderate }) \\
\text { T }(20-40 \text { million )=26 }\end{array}$ & 15 & 8 & 3 \\
\hline $\begin{array}{l}\text { Olegospermia (Weak }) \\
((1-20 \text { million) }=73\end{array}$ & 42 & 20 & 11 \\
\hline Azospermia (no sperm) $=20$ & Zero & Zero & Zero \\
\hline
\end{tabular}

Table (5): Number of Seminal Fluid Infection in relation to Sperm Density and Percentage (500 Sample).

Tested .:

\begin{tabular}{|l|c|c|c|c||}
\hline \multicolumn{1}{|c|}{ Sperm } & $\begin{array}{c}\text { Sperm Density } \\
\left(\times 10^{6} \text { cell/ml) }\right.\end{array}$ & No.of patients & No.of Infected & Percentage(\%) \\
\hline Normospermia & $>20$ & 209 & 31 & $14.8 \%$ \\
\hline Oligospermia & $2-19$ & 145 & 51 & $35.2 \%$ \\
\hline Oligospermia & $<2.0$ & 59 & 26 & $44.1 \%$ \\
\hline Azoospermia & $\mathrm{NIL}$ & 87 & 66 & $75.8 \%$ \\
\hline Total & & 500 & 174 & $34.8 \%$ \\
\hline
\end{tabular}

Pathogenic Organisms Isolated From Seminal Fluid :( 68 pure Culture )

Table (6) Identification and percentage of bacteria as etiological agents of seminal tract infection:-

\begin{tabular}{||c|c|c||}
\hline \multicolumn{1}{|c|}{ Organism } & No. of isolates & Percentage of isolates \\
\hline 1)Gram negative microorganism & 31 & $45.6 \%$ \\
\hline Escherichia coli & 3 & $4.4 \%$ \\
Proteus vuligars & 3 & $4.4 \%$ \\
Pseudomonas aeruginosa & & $23.5 \%$ \\
2)Gram positive microorganism & 16 & $2.9 \%$ \\
Streptococcus faecalis & 2 & $14.7 \%$ \\
Streptococcus pyogenes & 10 & $4.4 \%$ \\
Staphylococcus aureus & 3 & \\
Staphylococcus epidrmids & & \\
\hline
\end{tabular}


Table( 8) MBC ( Minimal bactericidal Concentration ) of antimicrobial agents

Antimicrobial agent : $\mathrm{MBC}(\mu \mathrm{g} / \mathrm{ml})$
\begin{tabular}{|l|ll|}
\hline Amoxycillin & 15 & $\mathrm{ug} / \mathrm{ml}$ \\
\hline Ciprofloxacin & 33 & $\mathrm{ug} / \mathrm{ml}$ \\
\hline Erythromycin & 10 & $\mathrm{ug} / \mathrm{ml}$ \\
\hline Gentamicin & 4 & $\mathrm{ug} / \mathrm{ml}$ \\
\hline Penicillin G & 10 & $\mathrm{ug} / \mathrm{ml}$ \\
\hline
\end{tabular}

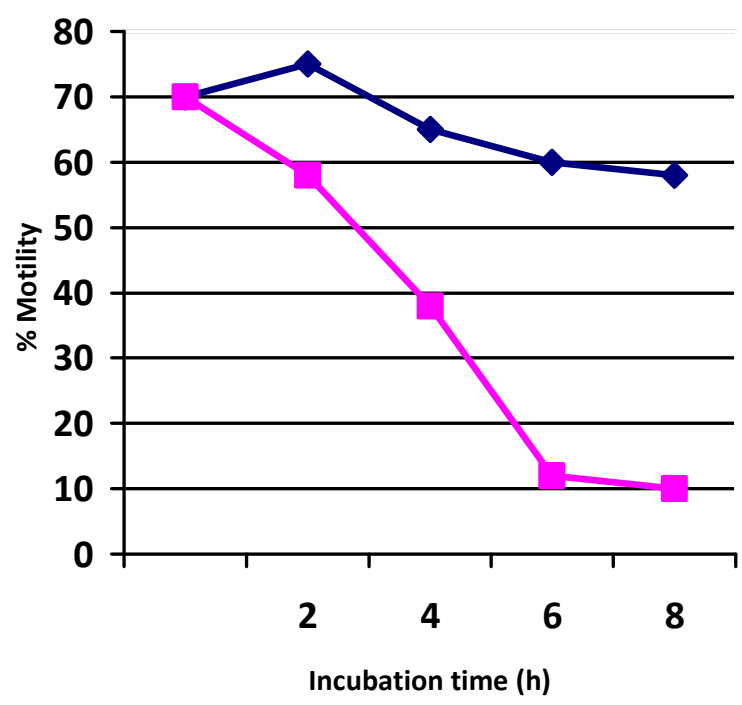

\section{DISCUSSION}

The present study demonstrated that human sperm motility and viability was affected by $\mathrm{E}$. coli. Also E. coli isolated from the semen of infertile male produced profound depression in the motility of human spermatozoa by agglutination in vitro. Schirren \& Zander (1966) in past reported a negative influence of $E$. coli on sperm motility after mixing sperm and bacteria in vitro. This negative influence was confirmed by several authors (Auroux, et al., 1991)., (Teague, et al., 1971) were the first to claim high numbers of bacteria as causative for this effect. After addition of antibiotics to the incubation medium, all bacterial effects on sperm motility disappeared. Bacterial growth may be one cause of contradictory conclusions concerning the effective sperm/ bacteria ratio. The absence of sperm agglutination was also observed after heat treatment of bacteria. In one of the earlier reports on effect of heat killed bacteria on spermatozoa characteristics (Kaur, et al., 2010) had studied that the live pathogenic S. aureus obtained from cervical cultures decreases in motility and viability of human spermatozoa which was absent in the sets

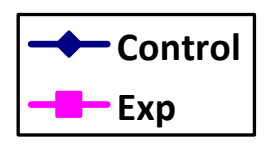

in which the bacteria had been killed by boiling for $30 \mathrm{~min}$ before mixing the ejaculate. Another study by (Hosseinzadeh et al., 2010) reported that elementary bodies of Chlamydia trachomatis Serovar E can induce premature sperm death upon co-incubation with human sperm but this effect is abolished when these EBs have been previously killed by heat treatment. Alternatively, effect of antimicrobial agents on bacteria was also observed. Treatment of cultured bacteria with different groups of antibiotics abolished the effect. This indicates that there must be some adhesion sites on spermatozoa to which E. coli binds. The mechanisms by which E. coli affect sperm functions have not yet been identified. Interference of E. coli with these receptors may influence the motility and viability. Other authors reported similar observations concerning sperm motility and agglutination after incubation with E. coli (Auroux, et al., 1991).

Thus, we suggest that E. coli/spermatozoainteraction may be a two-step process: i.e. adhesion to and subsequent destruction of the sperm membrane. This mechanism may account for any inhibitory effects of E. coli infection on 
male fertility. It is speculative whether the same mechanisms are also responsible for impaired male fertility in cases of genitourinary infection

\section{REFERENCES}

Ajabor L, Ezimokhai M and Kadiri A.(1999) Male contribution to subfertility in Benin City, Nigeria. Trop J Obst Gynaecol; 2:53.

Askienazy-Elbhar.(2005)Escherichiacoliattaches to human spermatozoa: affecting sperm parameters. Gynecol Obstet Fertil,; 33(9): 691-697.

Auroux MR, Jacques L, Mathieu D and Auer J.(1991). Escherichia coli attaches to human spermatozoa: affecting sperm parameters. Int J Androl, , 14:264-270.

Cheesbrough M. 1984 and 2007 ) Medical Laboratory Manual for Tropical Countries.VOL II Microbiology, EL BS Butterworth \& Co. Ltd, University Prees, Cambridge.

Comhaire F, Verschraegen $\mathrm{G}$ and Vermeulen L.(1980)Escherichia coli attaches to human spermatozoa: affecting sperm parameters. International Journal of andrology (); 3:32-45.

Dahlberg BI. (1976)Escherichia coli attaches to human spermatozoa: affecting sperm parameters. Urology, ; 8:563-66.

Hill JA, Haimovici F, Politch JA and Anderson DJ.( 1987) Escherichia coli attaches to human spermatozoa: affecting sperm parameters. Fertility and Sterility; 47:46065.

Hosseinzadeh S, Brewis IA, Eley A, Pacey AA, Moore HDM and Eley A. (2010) Escherichia coli attaches to human spermatozoa: affecting sperm parameters. Hum. Reprod,; 16:293-299.

Kaur S, Prabha V and Shukla G. (2010) Escherichia coli attaches to human spermatozoa: affecting sperm parameters.. Am J Biomed Sci,; 2(1): 91-97.

Keck C, Gerber-Schafer C, Clad A, Wihelm C and Breckwoldf M. (1998) Seminal tract infections: impact on male fertility and treatment options. Human Reproduction Update; 4(6): 891 - 903.

Mardh PA and Colleen S. (1975). Escherichia coli attaches to human spermatozoa: affecting sperm parameters. Scandinavian Journal of Urology and Nephrology; 9:17-23.

O'Leary W M. (1990) Escherichia coli attaches to human spermatozoa: affecting sperm parameters.. Cri. Rev. Microbiol,; 17(3): 161-168.

Panangala VS, WinterAJ, WijesinhaA and Foote RH.(1981). Escherichia coli attaches to human spermatozoa: affecting sperm parameters. Am. J. Vet. Res,; 42
Paulson JD. (1977) Escherichia coli attaches to human spermatozoa: affecting sperm parameters. Polakoski K.L., Fertil. Steril, 28:182-185.

Prabha V, Thakur N, Kaur S, Kaur N, Singh A and Kala S. (2009) Escherichia coli attaches to human spermatozoa: affecting sperm parameters. Am. J. Biomed. Sci,; $1(2): 126-132$.

Purvis K and Christiansen E. (1993) Infection in the male reproductive tract. Impact, diagnosis and treatment in relation to male infertility. Int J Androl;16:1-13.

Rowe PJ.. (2010) WHO Manual for the Standardized Investigation, Diagnosis and Management of the Infertile Male, 1st edn. Cambridge: Cambridge University Press.

Schirren C and Zander HA. (1966) Escherichia coli attaches to human spermatozoa: affecting sperm parameters. Medizini.qche Welt, , 45,45-47.

Scott AC. (1989) Laboratory control of antimicrobial therapy; In: Mackie and Mac Cartney Practical Medical Microbiology, Collee J G, Duguid J P, Fraser A G, Marmion B P (eds.) Churchill Livingstone UK Limited; 161-181.

Sergio C, Oehninger Thinus F and Kruger. (2007) Male Infertility Diagnosis and Treatment. Informa UK Ltd.; P: 345 .

Teague NS, Boyarsky S and Glenn JF. (1971) Escherichia coli attaches to human spermatozoa: affecting sperm parameters. Fertility and Sterility, ,22,281-85.

Tian YH, Liu J, Xu HM, Hu L and Xiong CL. (2005) Escherichia coli attaches to human spermatozoa: affecting sperm parameters. Zhonghua Nan Ke Xue,;11(3):179184.

Toth A and Lesser ML. (1981) Escherichia coli attaches to human spermatozoa: affecting sperm parameters. Fertil Steril, 36:88-91.

Weidner W, Jantos C, Schefer HG, Haidl G and Friedrich HJ. (1991) Escherichia coli attaches to human spermatozoa: affecting sperm parameters. Archives of Andrology,; 26:173-83

Wolff H, Panhans Stolz W and Meurer M. (1993) Escherichia coli attaches to human spermatozoa: affecting sperm parameters. Fertil Steril, 60(1), 154-158.

Wolff H, Politch JA, Martinez A, Haimovici E, Hill JA and Anderson D. J. (1990) Escherichia coli attaches to human spermatozoa: affecting sperm parameters. Fertility and Sterility, 53:528-36.

World Health Organization (1992), Laboratory Manual for the Examination of Human Semen and Sperm Cervical Mucus Interaction; 3d ed. Cambridge, UK, Cambridge Univ 